\title{
Le maître dans l'œil du disciple. À propos de l'apprentissage ou de l'initiation écologique dans Les Neuf consciences du Malfini de Patrick Chamoiseau
}

\section{Introduction}

Les Neuf consciences du Malfini de Patrick Chamoiseau se donne à lire comme un roman initiatique ou d'apprentissage écologique. Quoiqu'allégorique, il s'agit d'une biographie fictive centrée sur l'itinéraire d'un grand rapace carnassier adepte de la destruction de la faune de Rabuchon. Dans cette intrigue de maturation, le Malfini, le narrateur, s'attache à décrire les attitudes et les habitudes de son « infime maître » nommé le Foufou, qui l’ont fait passer de la prédation à la protection de l'environnement. Le récit est ponctué par la description des phases d'un apprentissage fondé sur l'observation minutieuse et l'imitation formatrice d'un modèle (le Foufou) faisant office de sujet écologique. Le comportement du Foufou vis-à-vis des autres composantes de la nature s'offre au Malfini comme une praxis écologique susceptible de l'aider à enterrer ses élans prédateurs et à décliner les mécanismes de sa renaissance symbolique. Cette réflexion se propose d'étudier le processus d'initiation du disciple et sa visée politico-écologique nécessaire à notre ère. Pour la conduire, nous aurons recours à l'écocritique qui «aborde le texte culturel selon une perspective politique qui décentre l'être humain pour mieux se recentrer sur l'environnement »

Paul Kana Nguetse - enseignant-chercheur au Département des Langues Étrangères Appliquées de l'Université de Dschang. Adresse de correspondance : Université de Dschang, Département des Langues Étrangères Appliquées, BP 49, Dschang, Cameroun ; e-mail : kanapaul83@yahoo.fr ORCID iD : https://orcid.org/0000-0002-2021-4175 
(Posthumus, 2014, p. 17). Étant donné que le disciple ou le maître est « une synthèse entre unités statiques (lêtre) et unités dynamiques (le faire)" (Jouve, 1992, p. 142), l'écocritique sera mise en perspective avec l'approche sémiologique du personnage de Philippe Hamon. Cette dernière permettra d'analyser, à partir des données fournies par le texte, l'identité profonde (la qualification et la fonction) du disciple en vue de montrer que son parcours initiatique rassemble un certain nombre "d'axes sémantiques fondamentaux pertinents " (Hamon, 1977, p. 129). Combinées, les grilles de lecture permettront de mettre en lumière la dynamique des relations maître-disciple, les procédés/le processus d'initiation et la modification de l'identité de l'initié dont la mise en texte infère des significations relatives à la pensée écologique.

\section{Rencontre du maître et mort symbolique du disciple}

Les Neuf consciences du Malfini épouse une structure tripartite représentative du roman initiatique : "Années de jeunesse »- " Années d’apprentissage » - "Années de maitrise ». Il " comporte une analogie structurale et symbolique suffisamment reconnaissable, précise et étroite [avec l'initiation religieuse] »(Vierne, 1987, p. 5). Dans cette section, il sera question d'analyser les années de jeunesse, c'est-à-dire les phases de préparation, de séparation et de qualification qui engendrent la mort symbolique du disciple.

Le roman de Chamoiseau est un récit allégorique, une parabole, une fable écologique dont les personnages sont des oiseaux. En dehors du statut animal des personnages qui sont d'ailleurs anthropomorphisés, il recèle toutes les caractéristiques du roman initiatique. En effet, cette œuvre met en scène un héros-narrateur qui fait le bilan de son passé, retrace les moments charnières de sa vie avant la rencontre de son maitre. Jeune, naïf et bourré d'idéaux, il fait face à des réalités qui tranchent avec ses croyances et ses convictions antérieures. De fait, une sorte de grand aigle nommé le Malfini raconte une rencontre qui a bouleversé sa vie d'indépendance dans la forêt de Rabuchon où il entretient des relations verticales avec les autres animaux. À l'orée du récit, il exalte ses prouesses, sa magnificence et avoue être le tout-puissant de ladite forêt : « Frère, vivant... ô Nocif... Je suis l'alarme. Je suis la toute-puissance. Je suis la peur et le danger. Rares sont les créatures qui, au bruit de mes ailes, ne se soient mises à geindre et à trembler » (Chamoiseau, 2009, p. 17). Dans cet extrait, il précise, dans un ton interpellatif et autoritaire, ses attributs qui se résument à la domination, à la fureur et à la prédation. En effet, le quotidien du Malfini est marqué par un agencement d'actions et de réactions souvent gratuites contre les autres espèces animales. Dans cette optique, il se présente comme l'être le plus respecté, le plus craint et le plus furieux de Rabuchon. Ainsi en témoigne-t-il :

J'avais en ce temps-là plaisir à foudroyer le cervelet d'une proie, à sentir sous mes serres l'immobilisation irréversible qui ouvre l'infini du néant. J'aime tuer. J'aime frap- 
per les chairs chaudes et me repaitre de la saveur du sang [...]. J'aime déchirer les muscles, éventrer, dérouler des boyaux, dissiper l'amertume d'une bile sous l'écrasement d'un foie... Et j'aime le tressautement de la chair qui abdique. (2009, p. 21)

Le Malfini est donc un oiseau carnassier qui se nourrit de la chair des autres animaux. Ses proies, précise-t-il, sont « des rats, des chauves-souris, des poules, des crabes, des grenouilles, des oiseaux vagabonds de bon sang et belle taille, des choses volantes ou pas, bien conséquentes et chaudes " (Chamoiseau, 2009, p. 22). Dans cette description de la prédation empreinte de délectation et d'innocence, il se dégage que le Malfini est un inconscient écologique jusqu'au moment où il fait la connaissance de son maitre. Dans sa posture de personnage en phase préparatoire d'initiation, il est, comme on vient de le voir, « caractérisé par sa jeunesse, par la mobilité de son caractère et par l'indétermination de ses projets. Doté d'une vision naïve du monde, il demande à être dirigé et guidé tout au long de l'itinéraire qui l'amène [...] à se fixer " (Demay \& Pernot, 1995, p. 94). Sa jeunesse est marquée par le fait que ses attitudes vis-à-vis de la faune ne sont pas à l'acmé d'un désir ; elles rentrent dans l'ordre normal des désirs organiques de son être. Il n'est donc pas conscient qu'il est sanguinaire et méchant parce que son comportement, à l'en croire, est « juste une force inscrite aux violences impassibles qui régentent le vivant» (Chamoiseau, 2009, p. 21). C'est sûrement pour cette raison qu'il conclut : « La vie était simple : mes chasses et mon repos. Le monde même était simple. D'un côté ce qui était utile à mon existence, de l'autre ce qui ne l'était pas. [...] Le monde était construit autour de moi, pour moi, avec comme seul aboutissement : le sang et la terreur» $(2009$, p. 22$)$.

$\mathrm{Au}$ regard de ce qui précède, la jeunesse et l'inconscience sont constitutives du profil psychologique de ce personnage qui se considère comme la perfection, la totalité ou le centre du monde. Sa vie et ses rapports aux autres animaux sont balisés par des convictions et la croyance qu'il est le tout-puissant, l'être suprême, l'animal au-dessus des autres animaux. C'est ainsi qu'il s'autorise à les chasser, les tuer et les dévorer. Son ignorance de l'interdépendance des constituants écosystémiques l'érige en un irresponsable écologique qui n'a pas fait sienne l'idée qu'il dépend de l'environnement naturel et réciproquement. Ainsi, il a une vision conservatrice et égocentrique de la nature. Il faut préciser que le disciple du Foufou évoque ce passé pour le regretter. Aussi confesse-t-il : « Je me croyais heureux, au faite d'une plénitude, avec comme ultime ambition l'envie de me vautrer au fond de ce bonheur. Rien à prouver, ni à moi-même ni à quiconque, terrible et tout-puissant, je n'éprouvais même plus la vanité de l'être » (2009, p. 19). Ici, le Malfini reconnaît que sa vie antérieure est une illusion de bonheur. La description des actes passés, doublée de l'aveu d'inconscience, rentre dans la purification psychologique et mentale du néophyte qui va entrer dans un nouvel univers. Par ailleurs, elle participe de la préparation du néophyte à « la mort initiatique » (Vierne, 1987, p. 16); laquelle contribue à la clarification son évolution. Il s'agit de sortir de la famille des prédateurs pour intégrer la sphère des protecteurs de la nature. L’aveu mélancolique contenu dans le précédent 
extrait apparaît comme l'amorce du processus de guérison et de transmutation ontologique inhérents au parcours de tout disciple.

Après cette phase préparatoire à l'initiation, la suite du roman retrace l'itinéraire du personnage principal qui, au détour d'une promenade, fait la rencontre d'un colibri minuscule appelé le Foufou. Pour préciser les circonstances de cette rencontre, il déclare :

C'était au temps de ma splendeur barbare. Je provenais de loin, me laissant transporter par ces vents de nulle part qui rabotent les îles de la mer Caraïbe [...]. Je n'espérais plus rien de ma vie, sinon la paix et le repos, et un peu de silence [...]. Ainsi, à peine avais-je survolé cet endroit qu'une buse hargneuse m’encombra le chemin. Une de ces rapacités ordinaires qui infestent le monde de leur être inutile. Sans doute le petit commandeur de ce lieu. (Chamoiseau, 2009, p. 20)

Le Foufou le fascine, le bouleverse et lui répugne par ses relations à la nature. Contrairement au Malfini qui se considère comme le maître, le centre ou le satrape de cette forêt, le Foufou vit comme une partie, un maillon, une composante de la faune et de la flore de Rabuchon. Au vu de la taille de son maître, le Malfini n'imagine pas qu'il est à même de le sortir de l'aveuglement et de la condescendance qui gouvernent ses actions. C'est pour cette raison qu'il tient un discours empreint de mépris et de dénigrement sur ce colibri. Aussi déclare-t-il : " Impossible de comprendre ce que c'était. Ça bougeait. Ça émettait un résidu de chaleur. Ça semblait être en vie mais n'avait à mes yeux aucun sens acceptable... Comment alors deviner que ce machin allait fasciner le reste de mon existence et m'élever à la grandeur pour toute l'éternité » (2009, p. 25). Il ressort de cet échantillon illustratif que le disciple, au contact de son maître, reste incrédule quant à ses aptitudes et à son efficacité. La fixation sur sa petitesse lui joue des tours au point où il le chosifie et le considère comme n’étant pas digne de lui : "Elle (la créature) n’était pas digne de moi, ni même digne de vivre... Je terrassai cette bêtise volante » (2009, p. 21). Toujours est-il que la découverte du Foufou, mais surtout de son mode d'action vis-à-vis de l'environnement naturel, incite le Malfini à le suivre quotidiennement afin de le comprendre et d'apprendre son être-au-monde/son faire écologique. C'est avec la rencontre des deux protagonistes qu'intervient la mort initiatique du disciple-Malfini. Il est clair avec Simone Vierne qu' « on ne peut pas modifier un état sans l'abolir au préalable" (Vierne, 1987, p. 16). Pour le cas d'espèce, la mort initiatique consiste en l'abandon de la prédation et à la récusation de la destruction des infrastructures environnementales. Pour la caractériser, Vierne retient deux traits :

Deux caractères surtout la marquent, qu’on peut trouver séparément ou de façon concomitante : la perte de connaissance, réelle ou simulée, et l'entrée impossible, du moins aux yeux de la raison et de l'expérience quotidienne, que l'on nomme souvent du nom qu'elle a pris dans la légende grecque de la Toison d'Or, les Symplégades. (1987, p. 17) 
En effet, on a affaire à un disciple stupéfait, ébahi et saisi d'un trouble. Dans le passage de la page vingt-cinq mentionné précédemment, il relève qu'à la première rencontre, le maître a suscité une incompréhension en lui. Progressivement, l'incompréhension provoque une fascination maladive, une hypnose morbide qui contredit ce qu'il a été, ce qu'il a pensé être ou ce qu'il a envisagé devenir. C'est pour cette raison qu'il dit : « je n'osais pas me formuler que moi, le puissant, membre de la lignée des aigles, j’étais fasciné par une bestiole dépourvue de toutes les qualités qui jusqu’alors m’avaient paru majeures » (Chamoiseau, 2009, p. 87). Il s'agit donc d'un disciple bouleversé, en proie à des troubles psychologiques suscités par l'identité et le mode de vie de son maitre. À ce sujet, il dit : « il y avait ce trouble... ce désir peut-être d'une autre perspective... un indicible appel. C'est alors qu'un hoquet s'empara de ma vie» (2009, p. 24). Ce que le Malfini appelle « le hoquet » s'apparente à ce que Vierne nomme "l'entrée impossible » c'est-à-dire la découverte d'une réalité diamétralement opposée à l'expérience quotidienne du néophyte. Dans une série d'interrogations rhétoriques, il remet sa vie antérieure en question et affirme sa détermination à suivre les « enseignements » du maître :

Combien de saisons n'avais-je pas poursuivi une vie apeurée ? Senti un os s'effondrer de mon bec ? Avalé l'irruption d'une belle giclée de sang ? Et... rien de tout cela ne me manquait. Je n'avais besoin de rien d'autre que de regarder ce petit être, de tenter de le comprendre, de deviner ce qu'il essayait, et... et de l'imiter comme cela métait possible et sans que j'en perçoive un début de raison (2009, p. 86).

Ce questionnement est l'indicateur majeur de la mort initiatique du Malfini et « constitue à la fois le terme de la préparation et le début de l'initiation » (Vierne, 1987, p. 14).

\section{Apprentissage et gestation symbolique du Malfini}

Dans ses relations avec le maitre, le Malfini s'approprie les expériences concrètes qui déclenchent son processus d'apprentissage. Lesdites expériences se résument en un ensemble de faits et gestes qui contribuent à la modification radicale de son statut. Cette partie ambitionne de montrer ces stratégies d'apprentissage et le processus de maturation ou de gestation du Malfini.

D’emblée, il faut relever que l'apprentissage de ce disciple prend trois saisons : « Je volai auprès de lui durant trois saisons de nuages, d'obscur et de lumière. Attentif au prodige de son vol, je ruminais le sentiment que mes ailes étaient bruyantes, lourdes, poussiéreuses, et je m'en lamentais à chaque contraction de mes terribles muscles " (Chamoiseau, 2009, p. 122). Pendant cette période près du maitre, il s'initie à la régénération et à la responsabilité environnementales. Le Foufou apprend au néophyte, à travers son « être » et son « faire ", à participer au vivant, à séveiller au monde environnant et à polliniser le monde. En guise de témoignage, le Malfini précise : «il (le Foufou) 
ne m’avait laissé aucune pratique, aucun rite, aucun enseignement, juste l'aptitude à contempler une fleur et le transport de graines et de poussières » $(2009$, p. 224). De ce passage, il ressort que dans leurs relations, il se transmet un savoir-être (la contemplation des fleurs) et un savoir-faire (le transport de graines et de poussières). Cette deuxième situation s'appelle la pollinisation, c'est-à-dire le déplacement des organes de reproduction mâle vers les organes de reproduction femelle, condition préalable de la reproduction sexuée des fleurs. Dans ce cas d'initiation, ce qui se passe

n'est ni objet du monde auquel on se référerait sur le mode de la désignation, ni concept (signification), ni enfin mode affectant le sujet de la tradition (manifestation). Quelque chose se passe qui ne peut se dire, mais seulement se montrer par approches ou circumbulations successives. (During, 1994, p. 407)

Ce mode d'initiation confère au Malfini le profil du disciple aristotélicien qui officie comme interprète et commentateur des gestes et des textes de son maître dont il délivre une lecture à partir de sa compréhension intime des pratiques magistrales. Il est à la quête de la libération de « son âme des contraintes corporelles pour la convertir, conversion sensée mener au divin » (Névot, 2013, p. 11). À l’inverse, et au regard du témoignage du Malfini, le Foufou peut être classé comme un personnage tutélaire que Demay et Pernot définissent comme suit : " personnage du roman d'apprentissage qui occupe une position d'autorité sur le personnage en formation auquel il adresse des discours tendant à lui expliquer les fonctionnements de la société dans laquelle il évolue de façon à lui indiquer comment y trouver sa place » (1995, p. 94).

Comme stratégie d'apprentissage, le Malfini coordonne ses allées et venues avec celles de son maître. Ainsi les décrit-il : « nous volions ensemble. Drôle de spectacle sans doute, que celui de ma majesté et de la fulgurance minuscule de mon maître, œuvrant ensemble à quelque chose de mystérieux " (Chamoiseau, 2009, p. 175). Le premier effet de l'initiation est la révision de ses rapports avec ses congénères. Ses relations sont désormais régies par le principe d'horizontalité et non de verticalité. L'horizontalité suppose qu'il faut considérer son vis-à-vis comme égal à soi, comme dépendant de soi et vice versa. Ce procédé d'apprentissage (le vol et le côtoiement pacifiques avec le Foufou) apparaît pour lui hilarant en ce sens qu'il n'entretenait, jusque-là, que des rapports conflictuels avec les autres animaux. Une autre stratégie est « la lenteur contemplative », l'imitation des stases contemplatives du maître qui revient à " voir fixe et longtemps, percevoir profondément, longtemps, veiller sans rien attendre, dans une haute vigilance » (2009, p. 83).

À la vérité, la relation maître-disciple cherche à instaurer un lien d'échange entre le formateur et l'apprenti. Or, pour le cas du Foufou et du Malfini, tout se passe comme s'ils faisaient du silence ou du mutisme la règle de l'apprentissage. " Rupture de la communication avec autrui et accession à la sainteté deviennent concomitantes, si bien que la pédagogie en tant que telle apparaît comme problématique, voire aporétique »(Levi, 1997, p. 52). Cette initiation s'apparente à l'enseignement 
taoïste de la Voie qui a pour principe pédagogique la dissolution de toute communication interactive. Dans le même sillage, le Malfini ajoute : "Bien que me tenant à distance, j'étais en osmose avec lui, je l'imitais intuitivement, percevais intuitivement ce qu'il faisait ou s'efforçait de faire, et je le reproduisais en moi-même sans attendre » (Chamoiseau, 2009, p. 83). Cependant, il faut mentionner que l'absence de tout échange verbal ou gestuel interpersonnel conscient entre les protagonistes est une forme de communication car, comme l'écrit Liezi Jishi à propos des situations pédagogiques taoïstes, «se servir du silence comme d'une parole, c'est encore parler ; faire de la non-connaissance un moyen de connaissance, c'est encore connaître ; ainsi donc, silence et non-parole, ignorance et nescience, tout cela est encore une manière de s'exprimer, une façon de connaître » (Jishi cité par Lévi, 1997, p. 53).

En tout état de cause, l'imitation du maitre provoque des chocs spirituels, des éveils à la connaissance et des orientations écologiques décisives chez le Malfini qui a eu la chance de le rencontrer et de le côtoyer. Aussi l'avoue-t-il : « Ma chance fut de rester auprès du petit maître » (Chamoiseau, 2009, p. 137). Auprès de ce dernier justement, il se rend compte de ses égarements, mieux, de son aveuglement. Il découvre un mode de vie, une posture existentielle, un « univers qu'il ne comprend pas et qu'il apprend peu à peu à connaître » (Aurégan, 1997, p. 56). C'est ainsi que ses analyses des actes, des actions et des réactions du Foufou lui permettent de revoir son statut et ses rapports à l'environnement. À titre illustratif, le Malfini affirme : "Dans son havre, je parvins à me convaincre qu'une opportunité métait offerte par ce nouveau danger : apprendre à dompter ma vision. Libérer mon regard de ces aveuglements que sont les habitudes... Il me fallut une demi saison de coït de rats pour découvrir comment " (Chamoiseau, 2009, p. 28). La libération des aveuglements est le point de départ d'une nouvelle vie marquée par le rejet de la prédation au profit de la protection de l'environnement. À la faveur de cette initiation, il s'opère une prise de conscience écologique. Celle-ci est donc le but de l'initiation qui est de permettre au disciple " de transcender son état, d'accéder à un statut radicalement différent de celui qui était auparavant le sien, étant bien entendu que, quels que soient les avantages sociaux qui peuvent découler éventuellement de ce nouvel état, ils ne sont pas l'essentiel » (Vierne, 1987, p. 66). Grâce à son maître, le Malfini se retrouve «à contempler des choses insignifiantes, donc ce qu'il y a de plus contraire à [lui], de plus éloigné, de plus répugnant ou répulsif pour [lui] !» (Chamoiseau, 2009, p. 86) pour parvenir au constat de l'inextricabilité des composantes de l'environnement naturel :

Je crus comprendre ce qui reliait ces existences : c’était la faim, la soif, la volonté de survivre et le désir farouche de se reproduire. Pour cela, animaux et végétaux s’emmêlaient selon les modalités infiniment complexes... Les existences des uns s'alliaient par la vie ou par la mort à l'existence des autres. La survie ou la mort des uns confortait celles de toute une série d'autres. [...] La présence des uns ralliait des milliers d'autres, qui eux-mêmes étaient des proies, des appâts et des nécessités pour des et-cætera d'autres... Un formidable commerce de vie et de morts qui se détruisaient en se construisant, qui se détruisaient en se construisant. (2009, p. 155-156) 
$\mathrm{Au}$ terme de cette section, quelques constats poétiques s'imposent et font de cette œuvre littéraire un roman initiatique archétypal. Alors que la plupart des romans d'apprentissage montrent des maitres qui sont des relais du savoir ou de la vérité par une mobilisation du dispositif multisensoriel et multimodal, celui-ci met le lecteur devant une situation "pédagogique " où la relation maître-disciple n'est pas interpersonnelle, interactive, volontaire ou ritualisée. Les scènes d'initiation sont contées par le disciple qui déroule les prouesses didactico-pédagogiques de son maitre. Dans ce sens, le Malfini argue que le Foufou ignore qu'il est son disciple : "Le Foufou ignora cette fièvre mimétique, comme s'il laissait chacun libre de faire ce qu'il voulait ou qu'il avait à faire " $(2009$, p. 205). De plus, l'objet de la formation n'est pas un enseignement véhiculé et reçu de gré à gré par le biais direct ou vivant de la parole ou de l'écoute. Nonobstant ces écarts esthétiques, l'apprentissage aboutit à la renaissance symbolique du Malfini.

\section{Renaissance symbolique du disciple et responsabilité écologique}

Dans cette partie, il sera question de mettre en lumière les indicateurs de la renaissance du disciple et de la glorification du maitre qui, en fin de compte, participent d'une stratégie d'interpellation des hommes sur la nécessité de protéger et de polliniser le monde. L'initiation du Malfini se termine par un harmonieux état d'équilibre, par une réconciliation avec l'environnement. Grâce à l'autorité de son maître, il est désormais en harmonie avec sa conscience, avec la nature et surtout avec ses congénères qu'il a méprisés jusque-là. En d'autres termes, il devient éco-protecteur comme le Foufou.

De prime abord, le but de l'initiation est de persuader le Malfini que tous les éléments de la nature se tiennent et se valent. Il s'agit de le pousser à comprendre que les relations entre les composantes doivent être horizontales et non pas verticales. Au terme de sa formation, il se réjouit de ne plus être le prédateur. C'est donc un disciple heureux qui ne voile pas son euphorie à la fin de son parcours initiatique. À ce propos, il déclare :

Je voyais tout différemment. Ces vies colorées, chatoyantes. Ces moires et ces velours qui participaient des états de la vie. Fasciné. Éclaté au plus large. Je passai des saisons à observer les vies de Rabuchon rien que pour les voir vivre. Rien que pour vivre d'elles [...] Comme j’avais été aveugle! Comme jétais hors du monde. Je compris encore mieux comment les vies se tiennent, combien nulle n'est centrale, plus digne, plus importante (Chamoiseau, 2009, p. 172).

Dans ce passage, le disciple décrit le changement radical de son statut, la modification de son mode de vie et la révision de ses rapports à l'environnement. En effet, 
ses activités ne sont plus la chasse et la prédation, mais la contemplation de la nature. Cette posture se justifie par l'interdépendance des constituants de la nature. En fait, il se rend à l'évidence qu'il (le Malfini) vit de ces derniers et réciproquement. Autant dire que protéger la nature, c'est se protéger, c'est protéger le vivant. S'appuyant sur ces constats, il parvient à la conclusion qu'aucun élément de la nature n'est central, plus digne et plus important que l'autre. Pour lui, « toute présence dans l'univers ou dans le multivers ouvre à la perspective d'une horizontale plénitude du vivant " (2009, p. 228). Le lecteur assiste ainsi à « la venue au monde d'un être nouveau, totalement différent de celui qui avait entrepris la périlleuse quête initiatique " (Vierne, 1987, p. 48). La série d'épreuves morales et physiologiques traversée à l'école du Foufou permet au disciple d'opérer une ascèse qui le mène du stade d'irresponsable écologique à celui de responsable écologique. Comme le récit est allégorique, il y a lieu de constater que le changement radical subi par le disciple est d'ordre ontologique. Cette ontologie transcendante s'observe sur les plans alimentaire et éthique.

Sur le plan nutritionnel, il se rend compte qu'il avait abusé du sang et de la chair d'autres espèces animales de cette forêt. Pour y remédier, il suspend ou réduit drastiquement la chasse. Le régime alimentaire change au point de se limiter à la simple hydratation. À ce titre, il atteste : "Je ne chassais presque plus, je ne mangeais que peu [...] N'éprouvais d'appétence que pour l'eau des orages que je gobais au vol. Ça me laissait du temps pour voir, contempler, admirer " (Chamoiseau, 2009, p. 174). En adoptant ce régime, il se rapproche de son maitre qui se nourrit essentiellement du nectar des fleurs. Néanmoins, la différence tient au fait qu'il ne peut pas pratiquer la technique du vol stationnaire qui permet au colibri de glisser son bec entre les pétales pour extraire le nectar. Comme on le lit dans cet extrait, le disciple prend très peu à la nature et lui donne plus. Conscient qu'en détruisant la nature il se consume, il devient très économique quant à l'exploitation des ressources de l'environnement. L'expérience du Foufou participe ainsi de la dissolution de certains conditionnements et des prédispositions à la prédation. Ladite expérience fonctionne comme une psychothérapie en ce sens qu'elle aide le disciple à changer de comportement et à s'adapter au réel. Elle semble réussie dans la mesure où l'évocation du triste passé provoque chez le Malfini répugnance et honte. Pour preuve, il avoue : « Je frémissais maintenant à l'idée de ces vies détruites par l'avidité carnassière de mon bec. Je tremblais de honte à l'idée de mon plaisir ancien sous chaque goulée de sang. Survivre mobilisait les accroches du plaisir, mais ces plaisirs pouvaient s'enfermer sur euxmêmes, vous retirer du monde» (2009, p. 174).

Sur le plan éthique et spirituel, il effectue une entrée salvatrice dans un au-delà sacré. Au même titre que tous les disciples, le Malfini vit à l'aune d'une éthique inculquée par le mystagogue. Il confesse d'ailleurs qu'il « demeure à chacun de [ses] gestes sous l'aube claire d'une éthique » (2009, p. 225). La transformation spirituelle du Malfini s’opère grâce à la conviction que toutes les présences vivantes de Rabuchon sont sacrées et méritent contemplation et protection. Elle s'articule également autour de l'idée que tout être reste fondamentalement tributaire de la nature c'est-à-dire 
des infrastructures écologiques de la planète, qui apportent des biens et des services essentiels à la subsistance. C'est pour cela qu'il reconnaît qu'il faut protéger son empreinte écologique ${ }^{1}$ en la pollinisant sans relâche. Dans ce sens, il affirme : «à la base de la vie, il y avait encore une infinité de possibles en devenir, de devenirs possibles... et qu'au fondement de toutes les vies de Rabuchon, [...] il y avait les fleurs » (2009, p. 172). À la vérité, en parlant des fleurs comme fondement de toute vie, l'auteur fait allusion à la pollinisation ; sorte d'activité naturelle menée par certaines espèces animales (les abeilles, les colibris, les chauves-souris...). En suçant le nectar des fleurs, elles y répandent des pollens transportés d'autres fleurs. Ce mode de reproduction favorise la croissance des plantes et la production des fruits nécessaires pour nourrir les hommes et les animaux. Conscient de ce fait, le Malfini s'évertue désormais à préserver la bonne santé de l'environnement. De fait, en s'appuyant sur l'idée que l'atteinte au vivant, sous quelque angle que ce soit, est une atteinte à l'ensemble de l'écosystème, il consacre le restant de son existence à la vénération et à la défense de l'équilibre écologique. Dans ce sens, le disciple du Foufou révèle : " Je devins d'une économie pieuse avec ce qui relevait de la vie» (2009, p. 174). Avec cette phrase, «la rencontre avec le sacré » (Eliade, 1992, p. 26), qui est l'objectif de toute initiation, se fait plus évidente. Elle se décline en un ensemble d'enseignements, de rites, d'épreuves que le Foufou mobilise et déploie pour « faire passer le néophyte de la vie infantile, [...] de la vie profane à la vie sacrée " (Eliade, 1992, p. 25). Ses activités dans la forêt sont désormais réglementées par l'idée du vivant. Bien que plus sensible au questionnement existentiel que par le passé, le Malfini éprouve une volupté ineffable à vivre sa nouvelle vie. Ainsi la décrit-il : « Ma vie [...] demeurait bien plus sensible qu’avant aux interrogations, mais j'en étais heureux : de ventée en ventée, je me réinstallais dans l'idée du vivant » (Chamoiseau, 2009, p. 175). Ce principe cardinal de son quotidien signifie que toutes les composantes de la nature s'équilibrent, se conditionnent, se forment, s'informent et se déforment en spirales interactives (voir 2009, p. 230). Sa conversion est source d'émulation et de bonheur. Cet état des choses lui permet de connaître et d'éprouver la joie de l'apprentissage et la félicité de l'initiation. Pour l'avoir initié à contempler les mystères et à se laisser porter par le sens du sacré (voir 2009 , p. 225), le disciple voue l'épilogue de son initiation à l'apologie et la glorification de son maitre. Il l'atteste dans l'échantillon ci-après : " Je m'envolai au-dessus du Foufou pour le remercier de ce qu'il m'avait apporté. Ce n'était plus un vol de majesté, mais l'étiquette d'une humilité reconnaissante. De respect aussi » (2009, p. 175). En plus de cette marque de gratitude, il reconnaît à son maître le statut de magicien parce qu' " il savait commander aux arcanes naturels, à ces insectes qui se mirent à peupler ce territoire ingrat. Il pouvait commander de même sorte aux rats, crabes, grenouilles, et à plein d'existences savoureuses qui sillonnaient partout, se poursuivant l'une l'autre» (2009, p. 154-155).

1. Par empreinte écologique, on entend la surface de la terre nécessaire pour assurer l'alimentation en eau et en vivres, le recyclage des déchets. 
En définitive, la situation de formation le Malfini/le Foufou correspond à la relation verticale qui existe entre la nature et l'homme qui se considère comme le centre ou le maître de l'univers. L'initiation du Malfini à la vénération et à la protection de la nature inverse le rapport, qui devient horizontal. Ce nouvel état induit que l'homme a à apprendre de la nature pour sa propre préservation. D'où la nécessité de l'horizontalité. Par cette allégorie, Patrick Chamoiseau remet en cause l'anthropocentrisme en tant que première cause du déclin écologique de notre ère. Ainsi, " [...] l'éthique se conjoint au poétique et au politique [...] le bonheur sensuel des mots exalte la beauté des paysages traversés, à travers l'inventaire précieux d'une Nature transformée en personnage » (Kassab-Charfi, 2012, p. 10). En outre, et à travers la modification du statut psychologique et existentiel du disciple à la fin du programme narratif, (renaissance du Malfini), le romancier préconise l'horizontalité dans les relations Homme/Nature, gage de l'équilibre écologique.

L'écriture [chamoisienne] permet [donc] de conscientiser le rapport au monde : présence intense, ardente, et en même temps retrait de l'écrivain qui se situe de loin, de haut, comme le Malfini, et qui butine la poussière d'or, comme le colibri, pour venir faire étinceler ses miroitements à nos yeux éblouis d'une telle virtuosité. (Desmaret, 2015, p. 25)

\section{Conclusion}

En définitive, Les Neuf consciences du Malfini est un récit d'initiation écologique, mais surtout une relation de l'expérience d'apprentissage d'un disciple. Il offre "l'image de l'homme en devenir » (Bakhtine, 1984, p. 227), qui constitue la caractéristique majeure du Bildungsroman. À rebours d'autres textes d'apprentissage qui se veulent la représentation des relations maitre-disciple, dans cette fable écologique, le lien pédagogique ne se déploie pas seulement entre les protagonistes mis en texte, mais aussi entre le roman et le « lecteur qui en constitue la cristallisation emblématique " (Levi, 2013, p. 63). D’ailleurs, dans le roman, le narrateur ne cesse d'apostropher le lecteur, de capter son attention comme pour vérifier la fluidité de la médiation ou la fidélité du récepteur. Le dénouement du texte fait office d'entame, mieux encore, de transition pour le lecteur appelé à passer de l'irresponsabilité à la responsabilité écologique. On peut alors dire avec Aurégan (1997, p. 60) qu'" au fond, le roman d'apprentissage est nécessairement inachevé, à l'image de la vie, ouvert sur une évolution laissée à l'appréciation du lecteur ». Somme toute, Les Neuf consciences du Malfini devient un guide de formation écologique, une praxis conative, une interpellation, du moins " une technique d'action sur le lecteur " (Hamon, 1981, p. 119). L'auteur interpelle ainsi l'homme sur le déclin écologique et l'invite à l'adoption d'une poétique rationnelle de la relation écologique. Celle-ci doit être fondée sur l'idée que toutes les composantes de l'environnement se tiennent, "se lient, se relient, se rallient, se relaient et se relatent " (Chamoiseau, 2009, p. 172). 


\section{RÉFÉRENCES}

Aurégan, P. (1997). Le Roman d'apprentissage au dix-neuvième siècle. Paris : Nathan.

Bakhtine, M. (1989). Esthétique de la création verbale. Paris : Gallimard.

Bancaud-Maenen, F. (1998). Le Roman de formation au dix-huitième siècle en Europe. Paris : Nathan.

Cailler, B. (2014). Poétique, politique et éthique de l'imaginaire dans Les Neuf consciences du Malfini de Patrick Chamoiseau. Présence africaine, 2(190), 285-295.

Chamoiseau, P. (2009). Les Neuf consciences du Malfini. Paris : Gallimard.

Demay, M.-C. et Denis, P. (1995). Le Roman d'apprentissage en France au XIXe siècle. Paris : Ellipses.

Eliade, M. (1992). Initiation, rites et sociétés secrètes. Naissances mystiques. Essai sur quelques types d'initiation. Paris : Gallimard.

Hamon, Ph. (1977). Pour un statut sémiologique du personnage. Dans R. Barthes, W. J. Kayser, W. C. Booth et Ph. Hamon (dir.), Poétique du récit (p. 115-180). Paris : Seuil.

Jouve, V. (1992). L'Effet-personnage dans le roman. Paris : Presses universitaires de France.

Kassab-Charfi, S. (2012). Patrick Chamoiseau. Paris : Gallimard / Institut français.

Levi, J. (2013). La transmission de la Voie de maître à disciples en Chine ancienne ou le Geste sans la Parole. Dans A. Névot (dir.), De l'un à l'autre. Maîtres et disciples (p. 35-63). Paris : CNRS éditions.

Névot, A. (2013). Au fil de la transmission. Un corps à corps maître-disciple. Dans A. Névot (dir.), De l'un à l'autre. Maîtres et disciples (p. 9-33). Paris : CNRS éditions.

Posthumus, S. (2014). Écocritique et ecocriticism. Repenser le personnage écologique. Figura, $36,15-33$.

Vierne, S. (1987). Rite, roman, initiation. Grenoble : Presses Universitaires de Grenoble.

Desmaret, M.-C. (2015). Phénoménologie et esthésie des images, des visions et des représentations dans la fable écologique de Patrick Chamoiseau Les Neuf consciences du Malfini. Apparition, disparition et réapparition, La Tortue Verte [Revue en ligne], 19-25. Récupéré de : http://www.latortueverte.com/Apparition Disparition Reapparition\%20 juillet 2015 la TortueVerte.pdf

RÉSUMÉ : Les Neuf consciences du Malfini de Patrick Chamoiseau est un roman initiatique articulé autour de l'expérience pédagogique d'un disciple et des prouesses didactico-pédagogiques d'un maître. Fondé sur l'observation minutieuse et l'imitation formatrice du maître, cet apprentissage permet à l'initié de transcender ses élans prédateurs pour accéder au stade de la responsabilité écologique. Contrairement au roman de formation traditionnel dans lequel le maître mobilise le dispositif multisensoriel et multimodal pour relayer le savoir ou la vérité, celui-ci met le lecteur devant une situation "pédagogique » archétypale où la relation maître-disciple n'est pas interpersonnelle, interactive, volontaire ou ritualisée. En outre, le lien pédagogique ne s'y déploie pas seulement entre les protagonistes mis en texte, mais aussi entre le roman et le lecteur qui en est la cible privilégiée. À l'aune de l'écocritique et de l'approche sémiologique du personnage, cette réflexion se propose d'étudier 
le processus d'initiation et la dynamique des relations maitre-disciple pour montrer que, par ce récit allégorique, Chamoiseau fait le procès de l'anthropocentrisme comme cause principale du déclin écologique et préconise l'horizontalité dans les relations Homme/Nature, gage de l'équilibre écologique.

Mots-clés : Chamoiseau, initiation, maître/disciple, écocritique, prédation, déclin écologique, responsabilité écologique

\section{The master in his disciple's eye(s). On learning or ecological education in Les Neuf Consciences du Malfini by Patrick Chamoiseau}

ABSTRACT: Les NeufConsciences du Malfini by Patrick Chamoiseau is a Bildungsroman centred around pedagogical experience of a disciple, and didactic and pedagogical achievements of a master. The educational process based on careful observation and formative imitation of the master, allows the novice to transcend his predatory impulses to reach the stage of ecological responsibility. Unlike the traditional Bildungsroman in which the master mobilizes some multisensory and multimodal devices to relay knowledge or truth, the novel in question confronts the reader with an archetypal "pedagogical" situation, where the master-disciple relationship is not interpersonal, interactive, voluntary or ritualized. Moreover, a pedagogical bond forms not only between the protagonists of the text, but also between the novel and the reader, its target audience. The paper proposes to approach the educational process and the master-disciple relationship from the point of view of ecocriticism, using semiotic analysis of a character. It is argued that by means of the allegorical account, Chamoiseau criticizes anthropocentrism for being the main cause of ecological degradation and advocates horizontality in the human-nature relationship, as a guarantee of ecological balance.

Keywords: Chamoiseau, initiation, master/disciple, ecocriticism, predatory behaviour, ecological degradation, ecological responsibility 